\title{
Soft Breaking of Gauge Invariance in Regularized Quantum Electrodynamics
}

\author{
T.R. Hurd *
}

Department of Mathematics, University of British Columbia, Vancouver, Canada, V6T 1Y4

\begin{abstract}
An alternative proof of the Ward-Takahashi identity for perturbative quantum electrodynamics is given which makes no use of a gauge invariant regularization such as the Pauli-Villars loop subtraction or dimensional regularization. Instead, it is shown, in the presence of an arbitrary high momentum cutoff $\Lambda$, that the exact W-T identity holds with an error of $O(1)$ $\Lambda^{-\varepsilon}$ with $0<\varepsilon<1$. The proof involves a perturbative analysis of the Euclidean functional integral for QED by the tree expansion method.
\end{abstract}

\section{Introduction}

A distinguishing feature of QED, and one which leads to considerable difficulties, is its gauge invariant character. The action for classical electrodynamics has a large symmetry group, known as the $U(1)$ gauge group, and this leads to the wellknown problem of a functional integral which is constant along orbits of infinite volume, and is hence infinite.

The standard method for handling this problem is known as gauge-fixing. An extra term is added to the action, which has the effect of introducing decay along the gauge orbits. The original gauge invariance is broken, but is replaced by a weaker invariance characterized by a functional equation known as the WardTakahashi identity. If the W-T identity can be demonstrated in the renormalized perturbation theory, various important questions can be answered. For example, it can be shown that the theory is perturbatively unitary and that the $S$-matrix is independent of the choices involved in gauge-fixing [7].

Thus, an essential part of the problem of perturbative renormalization of QED is to demonstrate the W-T identity appropriate to the choice of gauge-fixing. A formal derivation of the correct W-T identity can be obtained by a change of variables in the unrenormalized functional integral provided the action has no non-invariant terms other than the gauge-fixing term. The problem is to prove that the identity one arrives at in this way actually holds after renormalization.

* Research supported by the Natural Sciences and Engineering Research Council 
The first treatments of QED $[10,5,12,2]$ noted that non-invariant counterterms were apparently forbidden: This observation was made more concrete by the identities proved by Ward [13] and generalized by Takahashi [11]. Their idea evolved into what is now the standard solution of the problem $[1,3]$. Working in the presence of a gauge-invariant regularization for QED, we suppose inductively that no non-gauge-invariant counterterms have been introduced up to the $n^{\text {th }}$ order of perturbation theory. Then the W-T identity holds to order $n+1$, by the change of variables argument. But it can then be shown using this W-T identity that no non-invariant counterterms are needed in order $n+1$. Examples of suitable invariant regularizations are the Pauli-Villars loop subtraction [9] and dimensional regularization [7].

The proof of W-T identities presented here is quite different, in that no use is made of a gauge-invariant regularization. It is based instead on the following intuitively obvious idea. QED, if it is to be gauge invariant, should, when regularized in some arbitrary way by a cutoff with parameter $N$ and then renormalized appropriately, satisfy an identity of the form

$$
W^{N}+\delta W^{N}=0,
$$

where $W^{\infty}=0$ is the exact W-T identity and $\delta W^{N}$ goes to zero as the cutoff is removed. This should be true because the regularized theories uniformly approximate the non-cutoff theory. In fact, such a condition should be both necessary and sufficient for the theory to be gauge invariant. A proof along these lines has often been suggested, and is now feasible because of the development of a powerful method for bounding perturbation theory known as the renormalized tree expansion [6,3]. The proof of the W-T identity given in this paper involves two types of analysis: the first is a derivation of a functional identity of the form (1) for the theory with cutoff $N$, and the second is the detailed use of a variation of the tree expansion estimates to directly bound the error term $\delta W^{N}$.

In this paper, we consider Euclidean QED where, to avoid infra-red complications, we take a non-zero photon mass. By use of the method developed in [8], it should be possible to extend the present approach to the special case of zero mass photons and electrons. In Sect. 2, we state the W-T identity and indicate how it can be derived formally. A sequence of regularizations is chosen in Sect. 3, and the renormalized tree expansion estimates of [3] are reviewed. The approximate identity satisfied by the regularized theory is derived in Sect. 4, and a tree expansion for the error term is proved. This tree expansion is especially simple due to the special nature of the renormalization prescription adopted in Sect. 3 . Finally, in Sect. 5, tree expansion bounds are derived which show that the error term in the approximate W-T identity vanishes as the cutoff is removed. This is the main result of the paper.

The method developed in this paper should be applicable to pure Yang-Mills theory $\left(\mathrm{YM}_{4}\right)$, where it would yield a renormalization scheme without the need for dimensional regularization. Such a scheme has been developed as part of a constructive program by Feldman, Magnen, Rivasseau, and Sénéor [4], but their results, in particular for perturbation theory, are as yet unpublished.

This paper makes substantial use of the results of [3] and follows the notation set down there. 


\section{The Ward Identity}

Euclidean quantum electrodynamics involves a vector field $A_{\mu}$ (which we take with a non-zero mass) and spinor fields $\tilde{\psi}, \psi$ with the partition function

$$
\begin{aligned}
Z= & \int d A d \tilde{\psi} d \psi \exp \\
& -\int\left[\frac{1}{4} F_{\mu \nu} F^{\mu \nu}+\frac{1}{2}\left(\partial_{\mu} A_{\mu}\right)^{2}+\frac{1}{2} m_{p}^{2} A_{\mu} A^{\mu}+\tilde{\psi}\left(-i \not \partial+m_{e}+e \not A\right) \psi\right] .
\end{aligned}
$$

Here $(\partial \cdot A)^{2}$ is a gauge fixing term,

$$
\not A=\gamma^{\mu} A_{\mu}, \quad \not \partial=\gamma^{\mu} \partial_{\mu},
$$

where the $4 \times 4$ anti-hermitian Euclidean Dirac matrices satisfy

$$
\gamma^{\mu} \gamma^{\nu}+\gamma^{\nu} \gamma^{\mu}=-2 \delta^{\mu \nu}
$$

and $F_{\mu \nu}=\partial_{\mu} A_{\nu}-\partial_{\nu} A_{\mu}$ and $e$ is the bare electric charge. The free field photon covariance is $D^{\mu \nu}(x, y)=\delta^{\mu \nu}\left(-\partial^{2}+m_{p}^{2}\right)_{x y}^{-1}$ and the free field electron covariance is $S(x, y)=\left(-i \not \partial-m_{e}\right)_{x y}^{-1}$. For convenience in what follows, we suppose that the masses $m_{p}, m_{e}$ are $>1$.

Continuing in a formal way (i.e. by ignoring all questions of renormalization), we introduce the external effective potential $V_{e}\left(\Phi_{e}\right)$, which is the generating functional for the connected Green's functions amputated by the free field propagators, by the functional integral

$$
V_{e}\left(\Phi_{e}\right)=\log \int d P(\Phi) \exp (V+\delta V)\left(\Phi+\Phi_{e}\right) .
$$

If we now consider changes of variables $A=A^{\prime}, \psi=e^{i e \chi} \psi^{\prime}, \tilde{\psi}=e^{-i e x} \tilde{\psi}^{\prime}$ in (5) for arbitrary real scalar fields $\chi(x)$, we can arrive at the following Ward identity:

$$
\begin{aligned}
0=-\partial_{\mu} \frac{\partial V_{e}}{\delta A^{\mu}(x)} & -\int d y d x \frac{\delta V_{e}}{\delta \psi(x)}[i e \chi(x) \delta(x, y)+e S(x, y) \not \partial \chi(y)] \psi(y) \\
& +\int d y d x \tilde{\psi}(x)[i e \chi(x) \delta(x, y)+e \not \chi \chi(x) S(x, y)] \frac{\delta V_{e}}{\delta \tilde{\psi}(y)} \\
& +e \int d x: \tilde{\psi}(x) \not \chi \chi(x) \psi(x): .
\end{aligned}
$$

The non-linear form of this identity is

$$
\begin{aligned}
V_{e}(A, \psi, \tilde{\psi})= & V_{e}\left(A+\partial \chi,(1+e S \not \partial \chi) e^{-i e \chi} \psi, \tilde{\psi} e^{i e \chi}(1+e \not \partial \chi S)\right) \\
& +\int: \tilde{\psi} e^{i e \chi}\left(e \not \partial \chi+e^{2} \not \partial \chi S \not \partial \chi\right) e^{-i e \chi} \psi: .
\end{aligned}
$$

Even though the derivation here is formal, (7) is the identity we expect for the renormalized effective potential.

\section{Regularized QED}

We now review the renormalized tree expansion for the renormalized effective potential $V_{e}$. Following [3, Sect. 2], we introduce a logarithmic scale decomposition of the propagators

$$
D=\sum_{h=0}^{\infty} D^{(h)} ; \quad S=\sum_{h=0}^{\infty} S^{(h)}
$$


subject to bounds (for $M>1$ a fixed number)

$$
\begin{aligned}
& \left|\partial^{j} D^{(h)}(x)\right| \leqq O(1) M^{(2+|j|) h} \exp -M^{h}|x| \\
& \left|\partial^{j} S^{(h)}(x)\right| \leqq O(1) M^{(3+|j|) h} \exp -M^{h}|x| .
\end{aligned}
$$

The choice of scale decomposition is essentially arbitrary, but for concreteness we take the $\alpha$-parametric decomposition

$$
\begin{aligned}
& D^{(h)}(x)=(2 \pi)^{-1} \int_{I_{h}} d \alpha \alpha^{-2} \exp -\alpha m_{p}^{2}-(4 \alpha)^{-1} x^{2}, \\
& S^{(h)}(x)=(2 \pi)^{-1} \int_{I_{h}} d \alpha \alpha^{-2}\left(i \not \partial+m_{e}\right) \exp -\alpha m_{e}^{2}-(4 \alpha)^{-1} x^{2},
\end{aligned}
$$

where

$$
I_{h}= \begin{cases}{\left[M^{-2 h}, M^{-2 h+2}\right)} & \text { if } h>0 \\ {[1, \infty)} & \text { if } h=0 .\end{cases}
$$

For each integer $N>0$, the regularized free field propagators and measure are

$$
\begin{gathered}
D_{N}=\sum_{h=0}^{N} D^{(h)}, \quad S_{N}=\sum_{h=0}^{N} S^{(h)}, \\
d P_{N}(\Phi)=d A d \psi d \tilde{\psi} \exp -\frac{1}{2}\left(A, D_{N}^{-1} A\right)-\left(\tilde{\psi}, S_{N}^{-1} \psi\right),
\end{gathered}
$$

where we use $(\cdot, \cdot)$ to denote the standard inner product on $L^{2}\left(\mathbf{R}^{4}\right)$.

The renormalized regularized effective potential has the functional integral

$$
V_{e}^{N}\left(\Phi_{e}\right)=\log \int d P_{N}(\Phi) \exp V_{N}^{N}\left(\Phi+\Phi_{e}\right) .
$$

Here the bare interaction $V_{N}^{N}$ is "local" and lies in the span of the following monomials:

$$
\begin{array}{ll}
V^{1}=\int: A \cdot A:, & V^{2}=\int: \tilde{\psi} \psi:, \\
V^{3}=\int: F \cdot F:, & V^{4}=\int: \tilde{\psi}(-i \not \partial) \psi:, \quad V^{5}=\int: \tilde{\psi} \not A \psi:, \\
V^{6}=\int:(\partial \cdot A)^{2}:, & V^{7}=\int:(A \cdot A)^{2}: .
\end{array}
$$

We write $V_{N}^{N}=V+\delta V^{N}$, where the unrenormalized part is $V=-e V^{5}$ and the counterterms are $\delta V^{N}=-\sum_{i=1}^{7} \lambda^{i, N} V^{i}$. The counterterms are now completely determined by the renormalization condition

$$
L V_{e}^{N}=-e V^{5}
$$

where $L$, the localization operator, projects functionals of $\Phi$ onto the span of the monomials $V^{i}, i=1, \ldots, 7$. We will see the real reason for the particular choice (20) in Sect. 4.

The renormalized tree expansion for $V_{e}^{N}$ is

$$
V_{e}^{N}\left(\Phi_{e}\right)=V\left(\Phi_{e}\right)+\sum_{\substack{\text { trees } \tau \\ \tau \text { nontrivial }}} \frac{1}{n(\tau)} \sum_{\varrho} \sum_{\mathbf{h}} \mathscr{V}\left(\tau, \varrho, \mathbf{h}, \Phi_{e}\right),
$$


where $\varrho$ is a choice of the label $R_{f}$ of $C_{f}$ for each fork $f \in \mathscr{F}(\tau)$ and $\mathbf{h}$ is a choice of scale label $h_{f}$ for each $f \in \mathscr{F}(\tau)$, subject to the ordering

$$
\begin{cases}0 \leqq h_{f} \leqq h_{\pi(f)} & \text { if } \varrho_{f}=C \\ h_{\pi(f)}<h_{f} \leqq N & \text { if } \varrho_{f}=R\end{cases}
$$

and $h_{\pi(F)}=-1$ if $F$ is the lowest fork of $\tau$. The value $\mathscr{V}(\tau, \varrho, \mathbf{h}, \Phi)$ is calculated as a sum over connected Feynman graphs $G$ whose vertices represent the interaction $-e \int: \tilde{\psi} \not A \psi$ : whose lines represent either $S^{\left(h_{f}\right)}$ or $D^{\left(h_{f}\right)}$ or $S_{h_{f}}$ or $D_{h_{f}}$, whose legs (external lines) represent fields $\Phi$, and where $\tau$ defines a connectedness property of $G$ with respect to hard lines.

The crucial fact about this expansion is the following bound on the contribution of a given graph $G$ to $\mathscr{V}(\tau, \varrho, \mathbf{h}, \Phi)$ :

$$
\|G\| \leqq O(1) \prod_{f \in \mathscr{F}(\tau)} M^{\left(h_{f}-h_{\pi(f)}\right) \delta\left(G_{f}\right)},
$$

where the renormalized degree of divergence has the property that

$$
\begin{cases}\delta\left(G_{f}\right) \leqq-1 & \text { if } \varrho_{f}=R \\ 0 \leqq \delta\left(G_{f}\right) \leqq 2 & \text { if } \varrho_{f}=C .\end{cases}
$$

Here $\|\cdot\|$ is a suitable measure of the size of the distributional kernel $G$ represents. One easily shows from (23) and (24) that the sum over $\mathbf{h}$ is bounded, uniformly in the cutoff $N$. Thus the UV limit

$$
V_{e}=\lim _{N \rightarrow \infty} V_{e}^{N}
$$

exists as a formal power series in $e$.

It is now our goal to prove that the W-T identity is satisfied by $V_{e}^{N}$, with an error which is $O\left(M^{-\varepsilon N}\right)$. It then follows that $V_{e}$ defined by (25) satisfies the exact W-T identity (7).

As a remark before proceeding, we note that these approximate $\mathrm{W}$-T identities do not imply that noninvariant counterterms are less divergent than their degrees indicate. In fact, they get large for large $N$ with their natural degree of divergence. The correct intuition is this: The large noninvariant counterterms introduced into $\delta V^{N}$ are necessary to approximately cancel invariance breaking terms caused by the noninvariant cutoff.

\section{The Identity for $V_{e}^{N}$}

We now fix the cutoff $N$ at some arbitrary value, and derive a functional identity for $V_{e}^{N}$. Many equivalent statements are possible, but the derivation here leads to the most manageable formula for comparison with the exact identity (7).

We follow the formal derivation of (7) as far as we are able, and retain the error terms which arise from the non-invariant cutoff, and the non-invariant counterterms in $\delta V^{N}$. In contrast to the formal derivation, however, all manipulations we do now are well-defined by virtue of the finite cutoff. Consider changing variables in the functional integral by

$$
\psi(x)=e^{i e \chi(x)} \psi^{\prime}(x), \quad \tilde{\psi}=e^{-i e \chi} \tilde{\psi}^{\prime}, \quad A=A^{\prime}
$$


for $\chi$ any (bounded) smooth scalar field. We note that

$$
V^{i}\left(\Phi+\Phi_{e}\right)= \begin{cases}V^{i}\left(A+A_{e}, \psi^{\prime}+e^{-i e \chi} \psi_{e}, \tilde{\psi}^{\prime}+e^{i e \chi} \tilde{\psi}_{e}\right) & i \neq 4 \\ V^{4}\left(\psi^{\prime}+e^{-i e \chi} \psi_{e}, \tilde{\psi}^{\prime}+e^{i e \chi} \tilde{\psi}_{e}\right) & \\ \quad+V^{5}\left(e \partial \chi, \psi^{\prime}+e^{-i e \chi} \psi_{e}, \psi^{\prime}+e^{i e \chi} \tilde{\psi}_{e}\right) & i=4\end{cases}
$$

and by a standard identity for gaussian measures

$$
d P_{N}(\Phi)=c_{N} d P_{N}\left(\Phi^{\prime}\right) \exp -\left[:\left(\tilde{\psi}^{\prime}, e^{-i e x} S_{N}^{-1} e^{i e x} \psi^{\prime}\right):+:\left(\tilde{\psi}^{\prime}, S_{N}^{-1} \psi^{\prime}\right):\right],
$$

where $c_{N}$ is a constant we ignore. If $N=\infty,\left[S_{N}^{-1}, e^{i e \chi}\right]=e \not \chi \chi$, so we write

$$
\begin{aligned}
d P_{N}(\Phi)= & d P_{N}\left(\Phi^{\prime}\right) \exp -:\left(\tilde{\psi}^{\prime}, e \not \partial \chi \psi^{\prime}\right): \\
& \times \exp -\left[:\left(\tilde{\psi}^{\prime}, e^{-i e \chi}\left[S_{N}^{-1}, e^{i e \chi}\right] \psi^{\prime}\right):+:\left(\tilde{\psi}^{\prime}, e \not \partial \chi \psi^{\prime}\right):\right] .
\end{aligned}
$$

Insertion of these expressions into (16) yields the identity

$$
\begin{aligned}
V_{e}^{N}\left(\Phi_{e}\right)= & \log \int d P_{N}\left(\Phi^{\prime}\right) \exp \left(V+\delta V^{N}\right)\left(A+A_{e}, \psi^{\prime}+e^{-i e \chi} \psi_{e}, \tilde{\psi}^{\prime}+e^{i e x} \tilde{\psi}_{e}\right) \\
& \times \exp -e \lambda^{4} V^{5}\left(\partial \chi, \psi^{\prime}+e^{-i e \chi} \psi_{e}, \tilde{\psi}^{\prime}+e^{i e \chi} \tilde{\psi}_{e}\right) \\
& \times \exp -e V^{5}\left(\partial \chi, \psi^{\prime}, \tilde{\psi}^{\prime}\right) \\
& \times \exp :\left(\tilde{\psi}^{\prime},\left(e^{-i e x}\left[S_{N}^{-1}, e^{i e \chi}\right]-e \not \partial \chi\right) \psi^{\prime}\right):
\end{aligned}
$$

In the formal derivation of (7), $e \lambda^{4}=\lambda^{5}, \delta V^{N}$ has no $V^{2}, V^{6}$ or $V^{7}$ part, and so the $V^{5}$ terms above can be shifted into $V+\delta V^{N}$. We do the same shift here:

$$
\begin{aligned}
V_{e}\left(\Phi_{e}\right)= & \log \int d P_{N}\left(\Phi^{\prime}\right)\left[\exp \left(V+\delta V^{N}\right)\left(A^{\prime}+A_{e}+\partial \chi, \psi^{\prime}+e^{-i e \chi} \psi_{e}, \tilde{\psi}^{\prime}+e^{i e \chi} \tilde{\psi}_{e}\right)\right. \\
& \left.\times \exp e\left[V^{5}\left(\partial \chi, \psi^{\prime}+e^{-i e \chi} \psi_{e}, \tilde{\psi}^{\prime}+e^{i e \chi} \psi_{e}\right)-V^{5}\left(\partial \chi, \psi^{\prime}, \tilde{\psi}^{\prime}\right)\right]\right] \\
\times & {\left[\exp -\left(e \lambda^{4}-\lambda^{5}\right) V^{5}\left(\partial \chi, \psi^{\prime}+e^{-i e \chi} \psi_{e}, \tilde{\psi}^{\prime}+e^{i e \chi} \tilde{\psi}_{e}\right)\right.} \\
\times & \exp \sum_{i=2,6,7} \lambda^{i}\left[V^{i}\left(A^{\prime}+A_{e}+\partial \chi, \psi^{\prime}+e^{-i e \chi} \psi_{e}, \tilde{\psi}^{\prime}+e^{i e \chi} \tilde{\psi}_{e}\right)\right. \\
& \left.\left.-V^{i}\left(A^{\prime}+A_{e}, \psi^{\prime}+e^{-i e \chi} \psi_{e}, \tilde{\psi}^{\prime}+e^{i e \chi} \tilde{\psi}_{e}\right)\right]\right] .
\end{aligned}
$$

Let $P^{(1)}$ denote the operator

$$
\int d x \chi(x)\left[\left.\frac{\delta}{\delta \chi(x)}\right|_{\chi=0}\right]
$$

which extracts linear $\chi$ terms. We have

$$
\begin{aligned}
0= & P^{(1)}\left[V_{e}^{N}\left(A_{e}+\partial \chi,\left(1-i e \chi+e S_{N} \not \partial \chi\right) \psi_{e}, \tilde{\psi}_{e}\left(1+i e \chi+e \not \chi \chi \chi S_{N}\right)\right)\right. \\
& \left.+\int: \tilde{\psi}_{e} e^{i e \chi}\left[e \not \chi \chi+e^{2} \not \partial \chi S_{N} \not \chi \chi\right] e^{-i e \chi} \psi_{e}:\right] \\
+P^{(1)}[ & \log \int d P_{N}(\Phi) \exp \left(V+\delta V^{N}\right)\left(\Phi+\Phi_{e}\right) \\
& \left.\times \exp \left[\Delta_{N}(\chi, \psi, \tilde{\psi})+\delta \Delta_{N}\left(\chi, \Phi+\Phi_{e}\right)\right]\right] \\
\equiv & W^{N}+\delta W^{N}
\end{aligned}
$$


where

and

$$
\begin{aligned}
\Delta(\chi, \psi, \tilde{\psi})= & -e \int: \tilde{\psi}(x)\left[i S_{N}^{-1}(x, y)[\chi(y)-\chi(x)]\right. \\
& -\not \partial \chi(x) \delta(x, y)] \psi(y): d x d y
\end{aligned}
$$

$$
\delta \Delta_{N}\left(\chi, \Phi+\Phi_{e}\right)=\left(\lambda^{4}-\lambda^{5}\right) \tilde{V}^{5}\left(\chi, \Phi+\Phi_{e}\right)+\sum_{i=2,6,7} \lambda^{i} \tilde{V}^{i}\left(\chi, \Phi+\Phi_{e}\right)
$$

with

$$
\begin{array}{llrl}
\tilde{V}^{2} & =2 \int: \partial \chi \cdot A:, & & \tilde{V}^{5}=\int: \tilde{\psi} \not \chi \chi \psi:, \\
\tilde{V}^{6}=2 \int:(\partial \cdot \partial \chi)(\partial \cdot A):, & & \tilde{V}^{7}=4 \int:(\partial \chi \cdot A)(A \cdot A): .
\end{array}
$$

The first term $W^{N}$ of (33) clearly converges to the right-hand side of the Ward identity (7) as $N \rightarrow \infty$. To prove (7) it is sufficient to produce an $O\left(M^{-\varepsilon N}\right)$ bound on the second term $\delta W^{N}$ of (33).

We can write $\delta W^{N}$ as a sum over unrenormalized Feynman graphs, constructed with $V$ and $\delta V$ vertices, and exactly one insertion of either $\Delta$ or $\delta \Delta$. As we shall now see, there is a simple renormalized expansion for $\delta W^{N}$, similar to the expansion (21) for $V_{e}$ except with one $V$-insertion replaced by a $\Delta$-insertion. In other words, we will show that the terms $\delta \Delta$ in (33) act as exact counterterms to renormalize all subgraphs which contain the $\Delta$-insertion.

Lemma 1. The local parts $L\left(W^{N}\right)$ and $L\left(\delta W^{N}\right)$ are both zero.

Proof. Following [3, Sect. 4], in particular [3, Lemma 4.4], where properties of the localization operator are discussed,

$$
\begin{aligned}
L[ & \left.W^{N}(\chi, A, \psi, \tilde{\psi})\right] \\
= & P^{(1)} \sum_{s=0}^{d} T_{s}\left[V_{e}^{N}\left(A+\partial \chi,\left(1-i e \chi+e S_{N} \not \partial \chi\right) \psi, \tilde{\psi}\left(1+i e \chi+e \not \partial \chi S_{N}\right)\right)\right. \\
& \left.+\int \tilde{\psi} e^{i e \chi}\left[e \not \partial \chi+e^{2} \not \partial \chi S_{N} \not \partial \chi\right] e^{-i e \chi} \psi\right] \\
= & P^{(1)} \sum_{s=0}^{d} T_{s}\left[\left(L V_{e}^{N}\right)\left(A+\partial \chi,\left(1-i e \chi+e S_{N} \not \partial \chi\right) \psi, \tilde{\psi}\left(1+i e \chi+e \not \partial \chi S_{N}\right)\right)\right. \\
& \left.+\int \tilde{\psi} e^{i e \chi}\left[e \not \partial \chi+e^{2} \not \partial \chi S_{N} \not \partial \chi\right] e^{-i e \chi} \psi_{e}\right] .
\end{aligned}
$$

The renormalization condition says $L V_{e}^{N}=-e V^{5}$, and by use of the argument which proves [3, Lemma 4.3], we find

$$
\begin{aligned}
L\left(W^{N}\right)=-L(\delta W) & =P^{(1)} \sum_{s=0}^{d} T_{s}\left[-e \int \tilde{\psi} \not A \psi-e \int \tilde{\psi} \not \partial \chi \psi+e \int \tilde{\psi} \not \partial \chi \psi\right] \\
& =0 .
\end{aligned}
$$

Corollary 2. The following renormalized tree expansion holds for $\delta W^{N}$ :

$$
\delta W^{N}=\left.\right|_{-1} ^{\Delta}+\sum_{\substack{\text { nontrivial } \\ \text { trees }}} \frac{1}{n(\tau)} \sum_{\substack{\varrho \\ \varrho_{F}=R}} \sum_{\substack{\mathrm{h} \in \mathscr{H}(\tau, \varrho) \\ h_{\pi(F)}=-1}} P^{(1)} \overbrace{-1}^{\tau}
$$


where exactly one endpoint of the tree $\tau$ represents a $\Delta$-insertion, and the remaining endpoints represent $V$-insertions.

Proof. In the proof [3, Theorem 2.3] of (21), the renormalized tree expansion, it was necessary to verify the following conditions on the family of effective potentials $\left\{V_{r}^{N}\right\}_{r=-1, \ldots, N}$ :

$$
\begin{gathered}
V_{N}^{N} \in \operatorname{span}\left\{V^{i}\right\}_{i=1, \ldots, 7}, \\
L V_{-1}=-e V^{5},
\end{gathered}
$$

and to prove an inductive step relating $V_{r-1}^{N}$ to $V_{r}^{N}$ for $r=0, \ldots, N$. The proof here is exactly parallel. From (33) we see that $\delta W_{N}^{N}=\Delta^{N}+\delta \Delta^{N}$ satisfies

$$
\delta W_{N}^{N} \in \Delta+\operatorname{span}\left\{\tilde{V}^{i}\right\}_{i=2,5,6,7}
$$

and Lemma 1 implies

$$
L \delta W_{-1}^{N}=0 .
$$

The inductive step relating $\delta W_{r-1}^{N}$ to $\delta W_{r}^{N}$ is

$$
\delta W_{r-1}^{N}=\delta W_{r}^{N}+\sum_{m=1}^{\infty}(m+1) \varepsilon_{(r)}^{T}\left(V_{r}^{N}, \ldots, V_{r}^{N}, \delta W_{r}^{N}\right),
$$

where the $m^{\text {th }}$ term involves the usual $m+1$-fold truncated expectation. This inductive step can easily be shown if $\delta W$ is defined by the tree expansion (39) with the root taken at an arbitrary scale $r$ rather than -1 . It only remains to show that

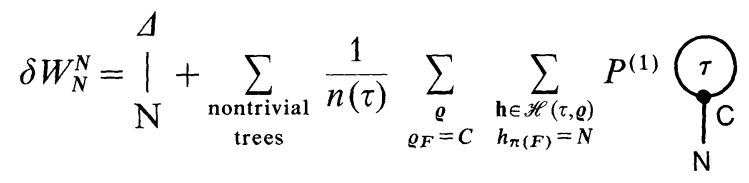

equals $\Delta$ plus local counterterms, verifying condition (40), and that the local part of the right-hand side of (39) is the local part of

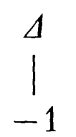

which vanishes because $\Delta$ is a Wick monomial depending on $\psi, \tilde{\psi}$ but not on $\psi_{e}$, $\tilde{\psi}_{e}$, verifying condition (41).

A priori, the renormalized expansion for $\delta W^{N}$ might have been much more complicated than this: the relatively simple expansion here exhibits exact renormalization cancellations because of the special renormalization condition (20) and the careful choices made in splitting (33) into two terms. Using (39) it is now possible to give a direct proof that $\delta W^{N}$ is $O\left(M^{-\varepsilon N}\right)$. Very roughly, it is clear that a $\Delta$-insertion into a Feynman graph will be $O\left(M^{-\varepsilon N}\right)$ if the graph is suitably convergent: the renormalized tree expansion has the effect of rendering all subgraphs suitably convergent. 
We now state the main result:

Theorem 3. Let $\widetilde{G}$ be a graph in the renormalized tree expansion for $\delta W^{N}$ associated with a tree $\tau$. Then for any $0<\varepsilon<1$ there exists a constant $c_{\varepsilon}(\widetilde{G})$ such that

Therefore

$$
\|\tilde{G}\| \leqq c_{\varepsilon}(\widetilde{G}) M^{-\varepsilon N} .
$$

$$
\lim _{N \rightarrow \infty} \delta W^{N}=0
$$

and $V_{e}$ satisfies the exact Ward identity (7).

\section{Proof of Theorem 3}

The renormalized tree expansion for $V_{e}^{N}$ and $\delta W^{N}$ differ by the replacement of exactly one vertex $V=-e \int: \tilde{\psi}(x) A_{e}(x) \psi(x)$ : by a vertex $\Delta$ which we write

where

$$
\Delta=-i e \int: \tilde{\psi}(x) \Delta_{N}(x, y)[\chi(x)-\chi(y)] \psi(y):,
$$

$$
\Delta_{N}(x, y) \equiv S_{N}^{-1}(x, y)-S^{-1}(x, y) .
$$

The kernel $\Delta_{N}$ goes to zero as $N \rightarrow \infty$ provided it is integrated against something sufficiently regular. But it turns out that the renormalized tree expansion ensures this regularity and it is possible to prove Theorem (3) by essentially pure power counting. We shall find a bound for an arbitrary graph $\widetilde{G}$ contributing to $\delta W^{N}$ by direct comparison to the bound

$$
\|G\| \leqq c_{0}^{l(G)} \sum_{\mathbf{h}} \prod_{f \in \mathscr{F}(\tau)} M^{\delta\left(G_{f}\right)\left(h_{f}-h_{\pi(f)}\right)}
$$

for a corresponding graph $G$ which contributes to $V_{e}^{N}$. Recall that in (48)

$$
\begin{cases}\delta\left(G_{f}\right) \leqq-1 & \text { if } \varrho_{f}=R \\ 0 \leqq \delta\left(G_{f}\right) \leqq 2 & \text { if } \varrho_{f}=C .\end{cases}
$$

and that $h_{\pi(F)}=0$ for the bottom fork of $\tau$.

The graph $G$ is obtained from $\widetilde{G}$ by the following "local" replacement

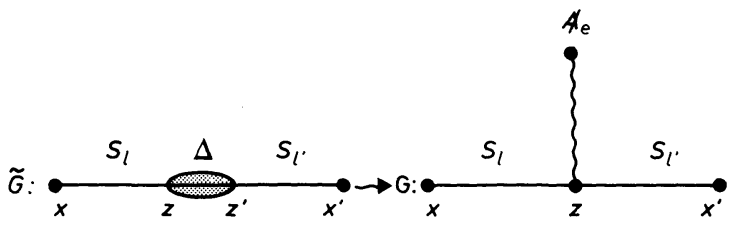

which we suppose occurs at the fork $f_{0}$. Let $\left\{f_{0}, f_{1}, \ldots, f_{n} \equiv F\right\}$ be the chain of forks hanging down from $f_{0}$ to $F$, and let their scales be $h_{i} \equiv h_{f_{i}}$.

In the unrenormalized case we write the value of $\tilde{G}$ and $G$ as follows:

$$
\begin{aligned}
\tilde{G}=-i e \int d x d x^{\prime} d z d z^{\prime} d \mathbf{y} S_{l}(x, z) \Delta_{N}\left(z, z^{\prime}\right)\left[\chi(z)-\chi\left(z^{\prime}\right)\right] S_{l^{\prime}}\left(z^{\prime}, x^{\prime}\right) \\
\times K\left(x, x^{\prime}, \mathbf{y}\right) \Pi\left(x, x^{\prime}, \mathbf{y}\right) \\
G=-e \int d x d x^{\prime} d z d \mathbf{y} S_{l}(x, z) \not A(z) S_{l^{\prime}}\left(z, x^{\prime}\right) \\
\times \\
\times K\left(x, x^{\prime}, \mathbf{y}\right) \Pi\left(x, x^{\prime}, \mathbf{y}\right),
\end{aligned}
$$

where the kernel $K$ and Wick monomial $\Pi$ are the same in both formulas. 
Note that due to the presence of the difference $\chi\left(z^{\prime}\right)-\chi(z)$ in $\Delta$, a zero ${ }^{\text {th }}$ order localization applied at any of the forks $f_{i}$ is zero. This fact is used to improve the $R$-operation for free at these forks: When the superficial degree is $\delta_{i} \leqq-1, R_{f_{i}}$ is defined to be a first-order Taylor subtraction instead of the identity operation. With this modification we can afford to treat the $\chi\left(z^{\prime}\right)$ and $\chi(z)$ terms of (51) separately.

Consider the term of $\tilde{G}$ containing $\chi\left(z^{\prime}\right)$ : After doing the $z$ integral we have

$$
\int R_{l N}\left(x, z^{\prime}\right) \chi\left(z^{\prime}\right) S_{l^{\prime}}\left(z^{\prime}, x^{\prime}\right) K\left(x, x^{\prime}, \mathbf{y}\right) \Pi\left(x, x^{\prime}, \mathbf{y}\right) d x d x^{\prime} d z^{\prime} d \mathbf{y},
$$

where

$$
R_{l N} \equiv S_{l} * \Delta_{N} .
$$

For a soft line $l$ of scale $h \leqq N$, the Fourier transform of $R$ is

$$
\hat{R}_{l N}(p)=\hat{R}_{N}^{<h}(p) \equiv\left[\exp -M^{-2(h-1)} p^{2}\right]\left[\exp M^{-2 N} p^{2}-1\right] .
$$

If $l$ is a hard line of scale $h<N$ then

$$
\hat{R}_{l N}(p)=\hat{R}_{N}^{(h)}(p) \equiv \hat{R}_{N}^{<h+1}(p)-\hat{R}_{N}^{<h}(p),
$$

but for $h=N$,

$$
\hat{R}_{N}^{(N)}(p)=1+\exp -M^{-2 N} p^{2}-\hat{R}_{N}^{<N}(p) .
$$

Taking care of the delta function in $R_{N}^{(N)}(x, y)$ requires some delicacy in the proof. It is easy to verify the following bounds

$$
\left|\partial_{x}^{n} R_{N}^{(h)}(x, y)\right| \leqq c_{n} M^{\varepsilon(h-N)} M^{(4+|n|) h} \exp -M^{h}|x-y|
$$

for any $0<\varepsilon \leqq 2$, where $\partial_{x}^{n}=\prod_{i=1}^{4}\left(\frac{\partial}{\partial x_{i}}\right)^{n_{i}}$ and $|n|=\sum_{i=1}^{4} n_{i}$. Of course, the delta function in $R_{N}^{(N)}$ does not satisfy (58) but the two other terms in $R_{N}^{(N)}$ do. We see that, except for the delta function in $R_{N}^{(N)}$, all $R$-lines have bounds consistent with standard power counting dimension $4=3+1$, times a decay factor $M^{\varepsilon(h-N)}$. The extra +1 in the dimension of $R$-lines compared to $S$-lines is to be expected if we think of $\Delta$ as a dimensionless vertex. This extra +1 is compensated by extra renormalization derivatives at the forks $f_{i}$ which occur because in the definition of localization at these forks, the exterior field $\chi$ has dimension 0 compared to 1 for $A_{e}$. We can immediately conclude that, except for a delta function term which occurs if $l$ is a hard $h=N$ line, (51) has the bound

$$
c c_{0}^{l(G)} M^{-\varepsilon N}\left[\sum_{\mathbf{h}} M^{\varepsilon h_{l}} \prod_{f} M^{\delta\left(\tilde{G}_{f}\right)\left(h_{f}-h_{\pi(f)}\right)}\right],
$$

where

$$
\begin{cases}\delta\left(\tilde{G}_{f}\right) \leqq-1 & \text { if } \varrho_{f}=R \\ 0 \leqq \delta\left(\tilde{G}_{f}\right) \leqq 3 & \text { if } \varrho_{f}=C .\end{cases}
$$

Now $h_{l}=h_{I}$ for some $0 \leqq I \leqq n$, and we distribute the factor $M^{\varepsilon h_{l}}$ down the tree from $f_{I}$ to $f_{n}$ :

$$
M^{\varepsilon h_{l}}=\prod_{i=I}^{n} M^{\varepsilon\left(h_{i}-h_{i+1}\right)},
$$


(where $h_{n+1} \equiv 0$ ). Since $\delta\left(\tilde{G}_{f}\right)+\varepsilon<0$ if $\varrho_{f}=R$, provided we take $0<\varepsilon<1$, (59) is bounded by

$$
O(1) M^{-\varepsilon N} \quad \text { for any } 0<\varepsilon<1 .
$$

When $l$ is a hard $h=N$ line, we still have the delta function term to worry about, which in the unrenormalized case looks like this

$$
\int \chi(x) S_{l^{\prime}}\left(x, x^{\prime}\right) K\left(x, x^{\prime}, \mathbf{y}\right) \Pi\left(x, x^{\prime}, \mathbf{y}\right) d x d x^{\prime} d z^{\prime} d \mathbf{y} .
$$

This corresponds to a graph with one fewer $V$-vertex than $G$, and with an extra external field $\chi$ attached at one vertex. Unfortunately, there is no completely trivial reason why this contribution is small for $N$ large, but in fact a factor $M^{-\varepsilon N}$ can be extracted. The only way to see this seems to be to analyse the different ways this term is renormalized.

The line $l$ enters at the fork $f_{I}$, and $h_{I}=N$. By the scale ordering on the tree $\tau$, all the branches emanating upward from $f_{I}$ join to endpoints or $C$-forks but not to $R$-forks, and thus the generalized vertices of the reduced graph $\tilde{g}_{f_{I}}$ are all local. Also by the scale ordering, we know that $f_{J}$, defined to be the highest $R$-fork with $J \geqq I$, has scale $N$.

Suppose $\tilde{g}_{f_{I}}$ has $v$ local vertices $v \geqq 3$, one of which is a $\Delta$-vertex or $\delta \Delta$ counterterm. The delta function contribution converts $\tilde{g}_{f_{I}}$ into a graph, call it $h_{f_{I}}$, with $v-1 \geqq 2$ vertices. We can think of $h_{f_{I}}$ as coming from a graph $H$ with standard power counting

$$
\sum_{\mathrm{h}} \prod_{f} M^{\delta\left(H_{f}\right)\left(h_{f}-h_{\pi(f)}\right)} .
$$

However, because the fork $f_{J}$ is at the fixed scale $N$, and $\delta\left(H_{f_{J}}\right) \leqq-1<-\varepsilon$, the factor $M^{\delta\left(H_{f_{J}}\right)\left(h_{J}-h_{J+1}\right)}$ can be replaced by $M^{-\varepsilon N} \prod_{i=J+1}^{n} M^{\varepsilon\left(h_{2}-h_{t}+1\right)}$. Just as before
the result is an $O(1) M^{-\varepsilon N}$ bound.

Suppose, finally, that there are exactly 2 local vertices feeding into $\tilde{g}_{f_{I}}$, one of which contains the $\Delta$-insertion. In this case, one can easily check that a Taylor derivative $T_{f_{I}}$ will always give zero for the term coming from the delta function in $R_{N}^{(N)}$. But we have argued that there is at least one Taylor derivative acting at $f_{I}$, whether $\varrho_{f_{I}}=R$ or $C$. Therefore, there is no contribution to $\widetilde{G}$ in this case, and we have proved the bound

$$
\|\tilde{G}\| \leqq O(1) M^{-\varepsilon N}
$$

for any $0<\varepsilon<1$.

Acknowledgements. It is a pleasure to thank Professor A. Wightman who raised the question in my presence, and to Professor L. Rosen, Professor J. Feldman and Dr. A. Cooper who made helpful suggestions during the preparation of this work.

\section{References}

1. Bogoliubov, N. N., Shirkov, D.V.: Introduction to the theory of quantized fields. New York: Wiley 1959

2. Dyson, F.J.: The radiation theories of Tomonaga, Schwinger, and Feynman. Phys. Rev. 75, 486-502 (1949) 
3. Feldman, J.S., Hurd, T.R., Rosen, L., Wright, J.D.: QED: a proof of renormalizability. Lecture Notes in Physics, Vol.312, Berlin, Heidelberg, New York: Springer, 1988

4. Feldman, J.S., Magnen, J., Rivasseau, V., Sénéor, R.: unpublished work (1989); and Sénéor, R.: Some remarks for the construction of Yang-Mills field theories, preprint, École Polytechnique, France (1988)

5. Feynman, R.P.: Space-time approach to quantum electrodynamics, Phys. Rev. 76, 769 (1949)

6. Gallavotti, G., Nicolò, F.: Renormalization theory in four-dimensional scalar fields, I and II, Commun. Math. Phys. 100, 545-590 (1985) and Commun. Math. Phys. 101, 247-282 (1986)

7. t'Hooft, G., Veltman, M.: Regularization and renormalization of gauge fields. Nucl. Phys. B44, 189-213 (1972) and, Combinatorics of gauge fields. Nucl. Phys. B50, 318-353 (1972)

8. Hurd, T.R.: A renormalization prescription for massless quantum electrodynamics. Commun. Math. Phys. in press (1989)

9. Pauli, W., Villars, F.: On the invariant regularization in relativistic quantum theory. Rev. Mod. Phys. 21, 434-444 (1949)

10. Schwinger, J.: On quantum electrodynamics and the magnetic moment of the electron. Phys. Rev. 73, 416 (1948) and, On radiative corrections to electron scattering. Phys. Rev. 75, 898 (1949)

11. Takahashi, Y.: On the generalized Ward identity. Nuovo Cimento 6, 370 (1957)

12. Tomonaga, S.: Infinite field reactions in quantum field theory. Phys. Rev. 74, 224 (1948)

13. Ward, J.C.: An identity in quantum electrodynamics. Phys. Rev. 78, 182 (1950)

Communicated by K. Gawedzki

Received January 16, 1989; in revised form March 20, 1989 